FACTA UNIVERSITATIS

Series: Mechanical Engineering Vol. 17, N 2, 2019, pp. 113 - 124

https://doi.org/10.22190/FUME190415018N

Original scientific paper

\title{
MECHANICAL STRUCTURE DESIGN TO AVOID FRICTION-INDUCED INSTABILITIES: IN-PLANE ANISOTROPY AND IN-PLANE ASYMMETRY
}

\author{
Ken Nakano ${ }^{1}$, Naohiro Kado ${ }^{1}$, Chiharu Tadokoro ${ }^{2}$, Takuo Nagamine ${ }^{2}$ \\ ${ }^{1}$ Yokohama National University, Yokohama, Japan \\ ${ }^{2}$ Saitama University, Saitama, Japan
}

\begin{abstract}
The stability of a two-degree-of-freedom (2DOF) sliding system with the velocity-weakening friction was examined by the eigenvalue analysis, where the inplane anisotropy and the in-plane asymmetry were considered. The obtained eigenvalues were organized by using the minimum modal damping ratio as the stability maps. Selecting a stable point in the stability map corresponds automatically to embedding the Yaw-Angle-Misalignment (YAM) method in the mechanical structure design to avoid the instability. If we accept the mechanical structure design of sliding systems with the in-plane anisotropy and the in-plane asymmetry, we can find new stable conditions spread widely in the two-dimensional space, which are invisible from the conventional point of view.
\end{abstract}

Key Words: Friction, Vibration, Instability, Stabilization, YAM Method

\section{INTRODUCTION}

Friction-induced instabilities are important problems to be solved since they result in vibration and noise of mechanical products (e.g., brakes, transmissions, wipers, belts, and tyres for automobiles). One of the most famous mechanisms of friction-induced instabilities is the velocity-weakening friction. It has been known that mechanical systems tend to become unstable when the frictional force decreases with decrease in the velocity [1] (e.g., the frictional force of lubricated sliding systems operating under mixed lubrication conditions).

Received April 15, 2019 / Accepted June 19, 2019

Corresponding author: Ken Nakano

Yokohama National University, Yokohama, Japan

E-mail: nakano@ynu.ac.jp 
When engineers have to solve the instability caused by the velocity-weakening friction, they always have two types of options. One is "improving frictional properties" and the other is "improving mechanical structures". Usually, they tend to choose the former since the former probably seems to be the lower-cost solution than the latter. However, in reality, a great deal of effort is required to find proper materials that show proper frictional properties. If they had some guidelines for mechanical structure design to avoid the instability in advance, the situations of the engineers would be improved.

Recently, as a promising method to stabilize mechanical systems suffering from the velocity-weakening friction, the "Yaw-Angle-Misalignment (YAM) method" has been proposed by the authors. The fundamental theory was first shown with an extended onedegree-of-freedom (1DOF) sliding system in the context of friction measurements [2]. In the fundamental theory, by observing the two velocities of the sliding surfaces in the "top view", their angular misalignment about the "yaw axis" was considered. After confirming the validity of the fundamental theory experimentally [2] and numerically [3], based on the structure of disc brakes, it was applied to a pad-on-disc-type sliding system numerically [4] and experimentally [5]. Besides, as another application format for rotational machines, the stabilizing effect of parallel misalignment in circular contacts was also shown [6].

From the viewpoint of guidelines for mechanical structure design, to examine the stabilizing effect in more practical sliding systems, the above fundamental theory was extended to a two-degree-of-freedom (2DOF) sliding system [7]. First, the stabilizing effect of the YAM method in the 2DOF sliding system was confirmed by numerical simulations. Then, the stability limit was examined by the eigenvalue analysis in a dimensionless form, which clarified the importance of the two quantities: the anisotropy of the in-plane stiffness (termed simply as the "in-plane anisotropy") and the asymmetry of the in-plane structure (termed simply as the "in-plane asymmetry"). However, as a price of using the dimensionless form, the final format did not become user friendly as a guideline for mechanical structure design.

In light of the above situation, in this study, the results of the eigenvalue analysis for the 2DOF sliding system are organized in a user-friendly format by focusing on the two most important quantities (i.e., the stiffness quantifying the "in-plane anisotropy" and the misalignment angle quantifying the "in-plane asymmetry") to embed the YAM method in mechanical structure design. By introducing the minimum modal damping ratio, the degree of stability is quantified and shown as the stability maps, which would be helpful for selecting the two quantities in the mechanical structure design of sliding systems.

\section{THEORY}

\subsection{Model}

Fig. 1 shows the analytical model, which is the 2DOF sliding system in the top view. A "ball" with mass $m$ is in contact with a "plate" parallel to the $x y$ plane at constant normal load $F_{z}$. The ball is supported elastically in the $x y$ plane by two springs with no damping. The stiffnesses in the $x$ and $y$ directions are $k_{x}$ and $k_{y}$, respectively. When $k_{x} \neq k_{y}$, they represent the "in-plane anisotropy" of the sliding system. The plate is driven at constant velocity $\mathbf{V}_{\text {A }}$ parallel to the $x y$ plane. Misalignment angle of $\mathbf{V}_{\text {A }}$ from the $x$ axis is 
$\varphi$. When $\varphi \neq 0$, it represents the "in-plane asymmetry" of the sliding system. Note that according to the above definition, the $x$ and $y$ axes are the principal axes of stiffness.

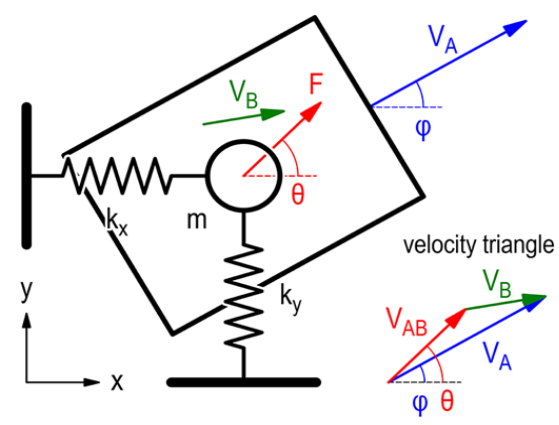

Fig. 1 Analytical model: 2DOF sliding system considering with "in-plane anisotropy" $\left(k_{x} \neq k_{y}\right)$ and "in-plane asymmetry" $(\varphi \neq 0)$

\subsection{Velocities}

Let $x$ and $y$ be the position of the ball (or the elongations of the two springs) as a function of the time $t$, i.e., $x=x(t)$ and $y=y(t)$. Relative velocity $\mathbf{V}_{\mathrm{AB}}$ of the plate to the ball is given by

$$
\mathbf{V}_{\mathrm{AB}}=\mathbf{V}_{\mathrm{A}}-\mathbf{V}_{\mathrm{B}},
$$

where $\mathbf{V}_{\mathrm{B}}$ is the instantaneous velocity of the ball. The magnitudes of $\mathbf{V}_{\mathrm{A}}$ and $\mathbf{V}_{\mathrm{B}}$ are given by

$$
\begin{aligned}
& V_{\mathrm{A}}=\left|\mathbf{V}_{\mathrm{A}}\right|=\text { constant }, \\
& V_{\mathrm{B}}=\left|\mathbf{V}_{\mathrm{B}}\right|=\sqrt{\dot{x}^{2}+\dot{y}^{2}},
\end{aligned}
$$

where (") is the derivative with respect to $t$. From the velocity triangle made by $\mathbf{V}_{\mathrm{A}}, \mathbf{V}_{\mathrm{B}}$, and $\mathbf{V}_{\mathrm{AB}}$, we obtain

$$
\begin{aligned}
& V_{\mathrm{AB}}=\left|\mathbf{V}_{\mathrm{AB}}\right|= \sqrt{\left(V_{\mathrm{A}} \cos \varphi-\dot{x}\right)^{2}+\left(V_{\mathrm{A}} \sin \varphi-\dot{y}\right)^{2}}, \\
& \sin \theta=\frac{V_{\mathrm{A}} \sin \varphi-\dot{y}}{V_{\mathrm{AB}}}, \\
& \cos \theta=\frac{V_{\mathrm{A}} \cos \varphi-\dot{x}}{V_{\mathrm{AB}}},
\end{aligned}
$$

where $\theta$ is the direction of $\mathbf{V}_{\mathrm{AB}}$, which is defined as the angle from the $x$-axis. Note that as shown in Fig. 1, $\theta$ is not necessarily equal to $\varphi$. 


\subsection{Equation of motion}

Let $\mathbf{F}=\mathbf{F}\left(\mathbf{V}_{\mathrm{AB}}\right)$ and $F=F\left(V_{\mathrm{AB}}\right)$ be the frictional force vector and its magnitude, respectively. Focusing on the ball, we obtain the following equation of motion:

$$
\mathbf{M} \ddot{\mathbf{x}}+\mathbf{K x}=\mathbf{F},
$$

where

$$
\begin{gathered}
\mathbf{x}=\left(\begin{array}{l}
x \\
y
\end{array}\right), \\
\mathbf{M}=\left(\begin{array}{cc}
m & 0 \\
0 & m
\end{array}\right), \\
\mathbf{K}=\left(\begin{array}{cc}
k_{x} & 0 \\
0 & k_{y}
\end{array}\right), \\
\mathbf{F}=\left(\begin{array}{c}
F\left(V_{\mathrm{AB}}\right) \cos \theta \\
F\left(V_{\mathrm{AB}}\right) \sin \theta
\end{array}\right) .
\end{gathered}
$$

Note that the direction of $\mathbf{F}$ is $\theta$ since it corresponds to the direction of $\mathbf{V}_{\mathrm{AB}}$. In steady sliding (i.e., $\mathbf{V}_{\mathrm{B}}=\mathbf{0}$ ), the ball remains at rest at the equilibrium position $\mathbf{x}=\mathbf{x}_{\mathrm{eq}}$ :

$$
\mathbf{x}_{\mathrm{eq}}=\left(\begin{array}{c}
\frac{F\left(V_{\mathrm{A}}\right) \cos \varphi}{k_{x}} \\
\frac{F\left(V_{\mathrm{A}}\right) \sin \varphi}{k_{y}}
\end{array}\right) .
$$

This is because $\mathbf{V}_{\mathrm{AB}}=\mathbf{V}_{\mathrm{A}}$ when $\mathbf{V}_{\mathrm{B}}=\mathbf{0}$, which leads to $V_{\mathrm{AB}}=V_{\mathrm{A}}$ and $\theta=\varphi$.

\subsection{Linearization}

Let $\tilde{\mathbf{x}}$ be the displacement disturbance from $\mathbf{x}_{\mathrm{eq}}$, that is:

$$
\tilde{\mathbf{x}}=\mathbf{x}-\mathbf{x}_{\mathrm{eq}}
$$

Substituting Eq. (13) into Eq. (7) and linearizing it around $\tilde{\mathbf{x}}=\mathbf{0}$ and $\dot{\tilde{\mathbf{x}}}=\mathbf{0}$ under the assumption that $\dot{\tilde{\mathbf{x}}} \ll \mathbf{V}_{\mathrm{A}}$, we obtain the following linearized equation:

$$
\mathbf{M} \ddot{\tilde{\mathbf{x}}}+\mathbf{C} \dot{\tilde{\mathbf{x}}}+\mathbf{K} \tilde{\mathbf{x}}=\mathbf{0},
$$

where

$$
\mathbf{C}=\left(\begin{array}{ll}
c_{x x} & c_{x y} \\
c_{y x} & c_{y y}
\end{array}\right) .
$$

The elements of $\mathbf{C}$ are given by

$$
c_{x x}=D_{1} \cos ^{2} \varphi+D_{2} \sin ^{2} \varphi,
$$




$$
\begin{gathered}
c_{x y}=c_{y x}=\left(D_{1}-D_{2}\right) \sin \varphi \cos \varphi, \\
c_{y y}=D_{1} \sin ^{2} \varphi+D_{2} \cos ^{2} \varphi,
\end{gathered}
$$

where

$$
\begin{aligned}
& D_{1}=F^{\prime}\left(V_{\mathrm{A}}\right), \\
& D_{2}=\frac{F\left(V_{\mathrm{A}}\right)}{V_{\mathrm{A}}} .
\end{aligned}
$$

Note that (') in Eq. (19) is the derivative with respect to $V_{\mathrm{AB}}$. Therefore, $D_{1}$ means the slope of the frictional force against the relative velocity at $V_{\mathrm{AB}}=V_{\mathrm{A}}$, which acts as a damper for the sliding system, although there are no dampers in the sliding system.

\subsection{Eigenvalue equation}

Using state vector $\mathbf{X}$ defined as

$$
\mathbf{X}=\left(\begin{array}{c}
\tilde{\mathbf{x}} \\
\dot{\tilde{\mathbf{x}}}
\end{array}\right),
$$

Eq. (14) is described as

$$
\dot{\mathbf{X}}=\mathbf{A X}
$$

where, by using zero matrix $\mathbf{O}$ and identity matrix $\mathbf{I}, \mathbf{A}$ is given by

$$
\mathbf{A}=\left(\begin{array}{cc}
\mathbf{O} & \mathbf{I} \\
-\mathbf{M}^{-1} \mathbf{K} & -\mathbf{M}^{-1} \mathbf{C}
\end{array}\right)
$$

Hence, by solving the following eigenvalue equation:

$$
\operatorname{det}(\mathbf{A}-s \mathbf{I})=0
$$

for $s$ :

$$
s=\sigma+j \omega
$$

the complex eigenvalues are obtained, where $j$ is the imaginary unit. If the real part $\sigma$ of every eigenvalue $s$ is negative, the equilibrium point is stable, which leads to steady sliding. Otherwise, the equilibrium point is unstable, which leads to friction-induced vibrations in the sliding system.

\section{METHOD}

The eigenvalue equation (Eq. (24)) was solved numerically under the assumption that

$$
F\left(V_{\mathrm{AB}}\right)=\mu_{\mathrm{k}}\left(V_{\mathrm{AB}}\right) F_{z},
$$

where $\mu_{\mathrm{k}}=\mu_{\mathrm{k}}\left(V_{\mathrm{AB}}\right)$ is the kinetic friction coefficient: 


$$
\mu_{\mathrm{k}}\left(V_{\mathrm{AB}}\right)=\mu_{\mathrm{k} \infty}+\left(\mu_{\mathrm{k} 0}-\mu_{\mathrm{k} \infty}\right) \exp \left(-\frac{V_{\mathrm{AB}}}{V_{\mathrm{f}}}\right),
$$

where $\mu_{\mathrm{k} 0}$ and $\mu_{\mathrm{k} \infty}$ are the dimensionless constants, and $V_{\mathrm{f}}$ is the velocity constant. In this study, the values of the three constants were $\mu_{\mathrm{k} 0}=2, \mu_{\mathrm{k} \infty}=1$, and $V_{\mathrm{f}}=10^{-2} \mathrm{~m} / \mathrm{s}$. Note that since $\mu_{\mathrm{k} 0}>\mu_{\mathrm{k} \infty}$, the magnitude of frictional force $F\left(V_{\mathrm{AB}}\right)$ shows the negative dependence on relative velocity $V_{\mathrm{AB}}$, which means the velocity-weakening friction.

\section{RESULTS AND DISCUSSION}

\subsection{Typical results}

Fig. 2 shows the real part $\sigma$ (upper) and imaginary part $\omega$ (lower) of every eigenvalue as functions of drive velocity $V_{\mathrm{A}}$. The left column (a) is for the "isotropic" stiffness $\left(k_{x}=k_{y}\right)$ and the "symmetric" structure $(\varphi=0)$. The middle column (b) is for the "anisotropic" stiffness $\left(k_{x} \neq k_{y}\right)$ and the "symmetric" structure $(\varphi=0)$. The right column (c) is for the "anisotropic" stiffness $\left(k_{x} \neq k_{y}\right)$ and the "asymmetric" structure $(\varphi \neq 0)$.
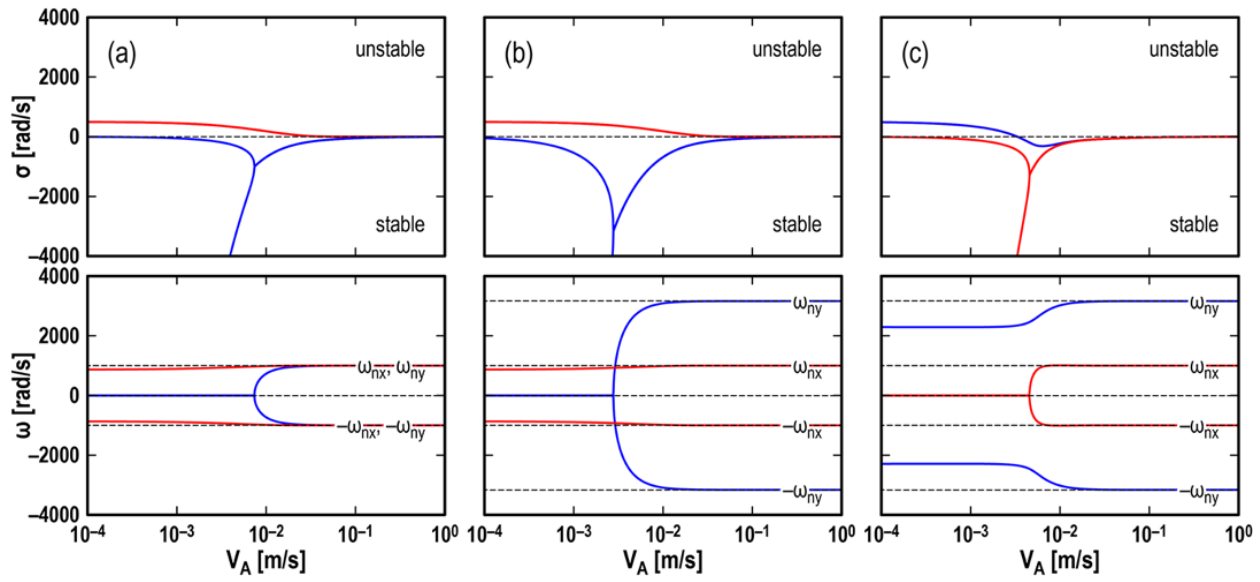

Fig. 2 Real part $\sigma$ (upper) and imaginary part $\omega$ (lower) of complex eigenvalue for $m=1 \mathrm{~kg}, k_{x}=10^{6} \mathrm{~N} / \mathrm{m}, \mu_{\mathrm{k} 0}=2, \mu_{\mathrm{k} \infty}=1, V_{\mathrm{f}}=10^{-2} \mathrm{~m} / \mathrm{s}$, and $F_{z}=10^{1} \mathrm{~N} ;$ (a) isotropic and symmetric $\left(k_{y}=10^{6} \mathrm{~N} / \mathrm{m}\right.$ and $\left.\varphi=0^{\circ}\right)$; (b) anisotropic and symmetric $\left(k_{y}=10^{7} \mathrm{~N} / \mathrm{m}\right.$ and $\left.\varphi=0^{\circ}\right) ;(\mathrm{c})$ anisotropic and asymmetric $\left(k_{y}=10^{7} \mathrm{~N} / \mathrm{m}\right.$ and $\left.\varphi=45^{\circ}\right)$

Four eigenvalues were obtained from the eigenvalue equation since the analyzed model was 2DOF. Some of them were conjugate: for example, the red curves of (a), the red curves of (b), and the blue curves of (c). Besides, for higher $V_{\mathrm{A}}\left(\right.$ e.g., $V_{\mathrm{A}}>10^{-2} \mathrm{~m} / \mathrm{s}$ ), the blue curves of (a), the blue curves of (b), and red curves of (c) were also conjugate.

Focusing on the upper graph of (a), the red curve locates above the zero line for any $V_{\mathrm{A}}$, which means that due to the velocity-weakening friction, the sliding system is always unstable. As shown in the upper graph of (b), this situation does not change even if the 
sliding system is anisotropic. However, as shown in the upper graph of (c), if the sliding system is not only anisotropic but also asymmetric, the blue curve changes from positive to negative with increase in $V_{\mathrm{A}}$. Considering that the sliding system cannot be asymmetric when it is isotropic, we can say that the combination of the in-plane anisotropy $\left(k_{x} \neq k_{y}\right)$ and the in-plane asymmetry $(\varphi \neq 0)$ is necessary to stabilize the sliding system. On the other hand, focusing on the lower graphs of (a) to (c), every curve is vertically symmetric about $\omega=0$. Note that the non-zero broken lines in the lower graphs show the natural frequencies defined as

$$
\begin{aligned}
& \omega_{\mathrm{n} x}=\sqrt{\frac{k_{x}}{m}}, \\
& \omega_{\mathrm{n} y}=\sqrt{\frac{k_{y}}{m}} .
\end{aligned}
$$
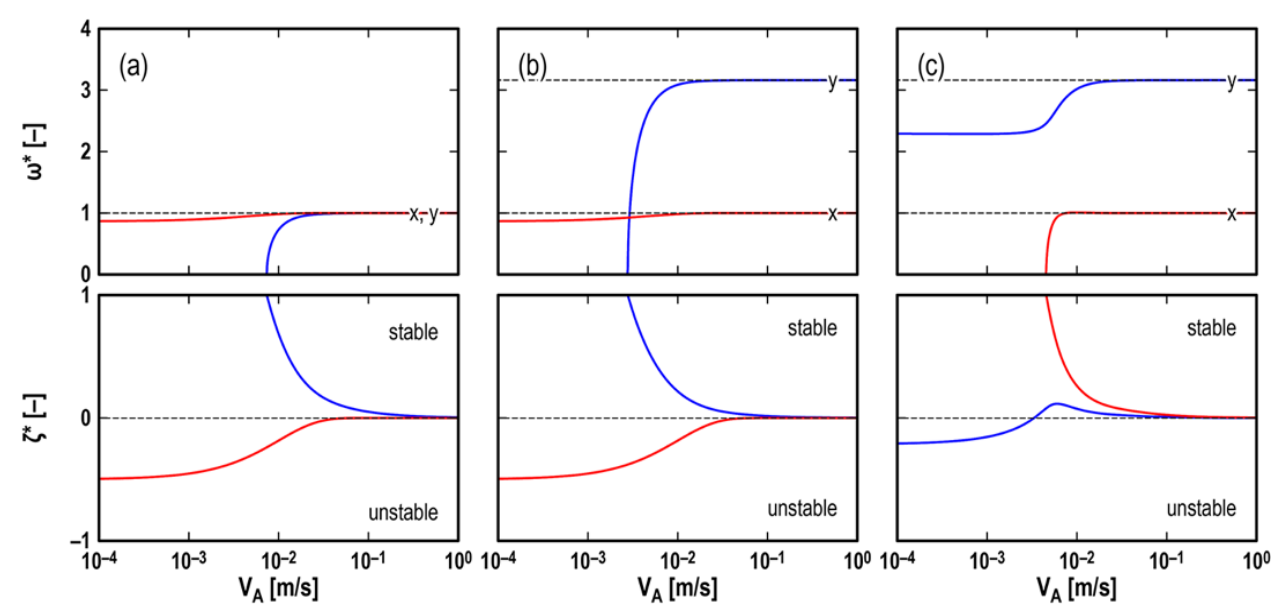

Fig. 3 Modal frequency ratio $\omega^{*}$ (upper) and modal damping ratio $\zeta^{*}$ (lower) for $m=1 \mathrm{~kg}$, $k_{x}=10^{6} \mathrm{~N} / \mathrm{m}, \mu_{\mathrm{k} 0}=2, \mu_{\mathrm{k} \infty}=1, V_{\mathrm{f}}=10^{-2} \mathrm{~m} / \mathrm{s}$, and $F_{z}=10^{1} \mathrm{~N} ;$ (a) isotropic and symmetric $\left(k_{y}=10^{6} \mathrm{~N} / \mathrm{m}\right.$ and $\left.\varphi=0^{\circ}\right)$; (b) anisotropic and symmetric $\left(k_{y}=10^{7} \mathrm{~N} / \mathrm{m}\right.$ and $\left.\varphi=0^{\circ}\right) ;(\mathrm{c})$ anisotropic and asymmetric $\left(k_{y}=10^{7} \mathrm{~N} / \mathrm{m}\right.$ and $\left.\varphi=45^{\circ}\right)$

Fig. 3 shows the same complex eigenvalues as Fig. 2, which are shown by using the modal frequency ratio $\omega^{*}$ and the modal damping ratio $\zeta^{*}$ defined as

$$
\begin{gathered}
\omega_{i}^{*}=\frac{\left|\omega_{i}\right|}{\omega_{\mathrm{n} x}}, \\
\zeta_{i}^{*}=-\frac{\sigma_{i}}{\sqrt{\sigma_{i}^{2}+\omega_{i}^{2}}},
\end{gathered}
$$


respectively. Note that by using $\omega^{*}$ and $\zeta^{*}$, four eigenvalues are found to be reduced to two values (i.e., red and blue). For the sliding system to be stable, every $\zeta^{*}$ needs to be positive, where $\zeta^{*}$ takes a value in the range of $-1 \leq \zeta^{*} \leq 1$.

Fig. 4 shows the effect of the misalignment angle $\varphi$ on $\omega^{*}$ and $\zeta^{*}$. Note that the $\omega^{*}$ and $\zeta^{*}$-values at $\varphi=45^{\circ}$ in Fig. 4 correspond to those values at $V_{\mathrm{A}}=10^{-2} \mathrm{~m} / \mathrm{s}$ in Fig. 3(c). When $\varphi<\varphi_{\mathrm{cr} 1} \sim 27^{\circ}$, the sliding system is unstable due to the negative $\zeta^{*}$ denoted by the red curve. On the other hand, when $\varphi>\varphi_{\mathrm{cr} 2} \sim 60^{\circ}$, the sliding system is also unstable due to the negative $\zeta^{*}$ denoted by the blue curve. However, only when $\varphi_{\mathrm{cr} 1}<\varphi<\varphi_{\mathrm{cr} 2}$, the both $\zeta^{*}$-values are positive, which means the sliding system is stable. As stated above, the combination of the in-plane anisotropy $\left(k_{x} \neq k_{y}\right)$ and the in-plane asymmetry $(\varphi \neq 0)$ is necessary to stabilizes the sliding system. However, it is not sufficient.

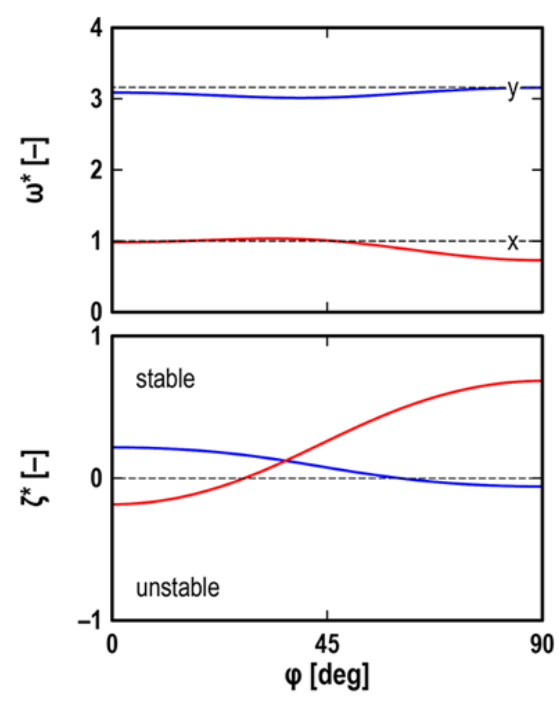

Fig. 4 Effect of in-plane angular misalignment $\varphi$ on modal frequency ratio $\omega^{*}$ (upper) and modal damping ratio $\zeta^{*}$ (lower) for $m=1 \mathrm{~kg}, k_{x}=10^{6} \mathrm{~N} / \mathrm{m}, k_{y}=10^{7} \mathrm{~N} / \mathrm{m}, \mu_{\mathrm{k} 0}=2$, $\mu_{\mathrm{k} \infty}=1, V_{\mathrm{f}}=10^{-2} \mathrm{~m} / \mathrm{s}, F_{z}=10^{1} \mathrm{~N}$, and $V_{\mathrm{A}}=10^{-2} \mathrm{~m} / \mathrm{s}$

Note that the strongest asymmetry does not necessarily mean $\varphi=45^{\circ}$. As shown in the lower graph of Fig. 4 , the red curve crosses with the blue curve at $\varphi=\varphi_{\text {opt }} \sim 36^{\circ}$. By considering that the stability of the sliding system is governed by the minimum modal damping ratio:

$$
\zeta_{\min }^{*}=\min \left(\zeta_{i}^{*}\right)
$$

$\varphi=\varphi_{\text {opt }}$ is believed to be a favorable choice for the mechanical structure design since $\zeta^{*}$ min takes the maximum at $\varphi=\varphi_{\text {opt }}$. In light of the above discussion, in the next section, the results of the eigenvalue analysis are shown by using $\zeta^{*}$ min as the stability maps. 


\subsection{Stability maps}

Fig. 5 shows the stability maps in the $k_{y}-V_{\mathrm{A}}$ plane. The value of $\zeta^{*}{ }_{\text {min }}$ from -1 to 1 is shown by the color from red (unstable) to blue (stable), respectively. The horizontal broken line in each graph shows the "isotropic" condition (i.e., $k_{x}=k_{y}$ ). The left and right graphs are for the "symmetric" conditions (i.e., $\varphi=0^{\circ}$ and $90^{\circ}$, respectively), while the middle graph is for the "asymmetric" condition (i.e., $\varphi=45^{\circ}$ ).

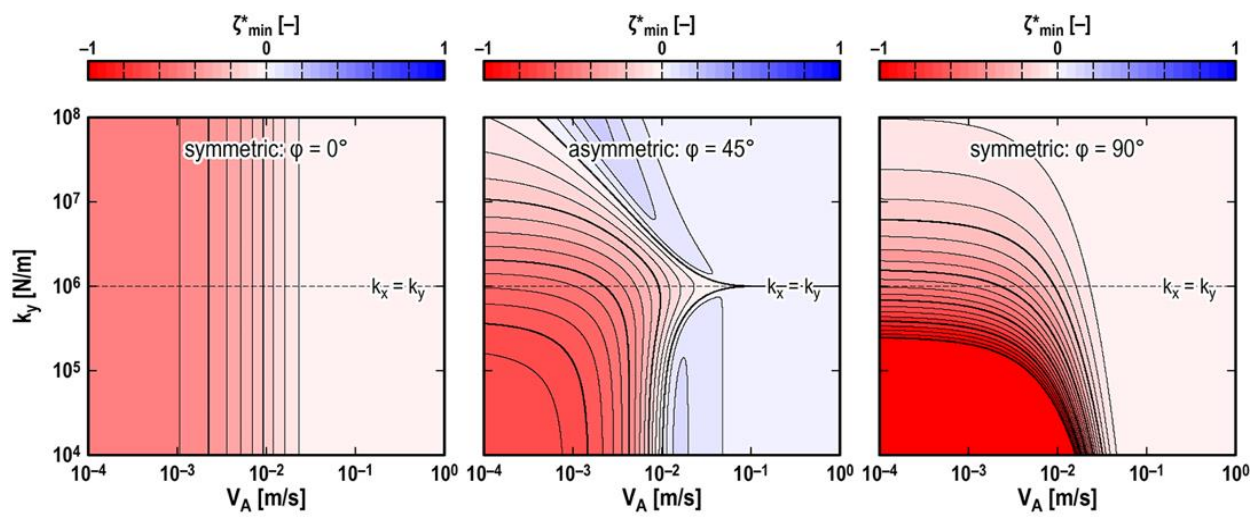

Fig. 5 Stability maps in $k_{y}-V_{\mathrm{A}}$ plane with minimum modal damping ratio $\zeta^{*}{ }_{\min } ; m=1 \mathrm{~kg}$, $k_{x}=10^{6} \mathrm{~N} / \mathrm{m}, \mu_{\mathrm{k} 0}=2, \mu_{\mathrm{k} \infty}=1, V_{\mathrm{f}}=10^{-2} \mathrm{~m} / \mathrm{s}$, and $F_{z}=10^{1} \mathrm{~N}$; red: unstable $\left(\zeta_{\min }^{*}<0\right)$ and blue: stable $\left(\zeta^{*}{ }_{\min }>0\right)$

The blue color $\left(\zeta^{*} \min >0\right.$ : stable) can be seen only in the middle graph, which locates above $\left(k_{y}>k_{x}\right)$ and below $\left(k_{y}<k_{x}\right)$ the horizontal broken line. This means that if the sliding system is asymmetric, not only increasing the stiffness but also decreasing the stiffness is effective for stabilization. However, comparing the upper blue portion with the lower blue portion, the stability limit of the former is wider than the latter. The stability limit for the upper blue portion seems to have a slope of -1 for lower $V_{\mathrm{A}}$, while the stability limit for the lower blue portion seems to be determined by $V_{\mathrm{A}}=V_{\mathrm{f}}=10^{-2} \mathrm{~m} / \mathrm{s}$. This indicates that increasing the higher stiffness $\left(k_{y}>k_{x}\right)$ is better than decreasing the lower stiffness $\left(k_{y}<k_{x}\right)$ although both these changes strengthen the in-plane anisotropy.

Note that the red color $\left(\zeta^{*}{ }_{\min }<0\right.$ : unstable) locating for lower $V_{\mathrm{A}}$ is caused by the provided frictional property (see Eq. (27)). The velocity-weakening friction $\left(\mu_{\mathrm{k} 0}>\mu_{\mathrm{k} \propto \infty}\right)$ makes the damping coefficient $D_{1}$ negative (see Eq. (19)), the value of which is decreased by decreasing $V_{\mathrm{A}}$, which enhances the instability for lower $V_{\mathrm{A}}$. The other damping coefficient $D_{2}$ is the essence of the YAM method, which is always positive (see Eq. (20)). When the sliding system is symmetric $(\varphi=0)$, the damping term of $D_{2}$ dissapears because $\sin ^{2} \varphi=0$ (see Eq. (16)). However, when the sliding system is asymmetric $(\varphi \neq 0)$, the positive $D_{2}$ becomes the competitor of the negative $D_{1}$ (see Eq. (16)). This is the fundamental mechanism of the YAM method to stabilize the sliding system, which is strongly related to the in-plane rotation of the frictional force vector $[8,9]$.

Fig. 6 shows the stability maps in the $k_{y}-\varphi$ plane, which consists of nine graphs. The bottom, middle, and top rows are for the normal load $F_{z}=10^{-1}, 10^{1}$, and $10^{3} \mathrm{~N}$, respectively. 
The left, middle, and right columns are for the drive velocity $V_{\mathrm{A}}=10^{-4}, 10^{-2}$, and $10^{0} \mathrm{~m} / \mathrm{s}$, respectively. The ordinate of each graph is stiffness $k_{y}$ representing the in-plane anisotropy of the sliding system, while the abscissa of each graph is misalignment angle $\varphi$ representing the in-plane asymmetry of the sliding system. The value of $\zeta^{*}$ min is shown by the color in the same manner as the previous figure.
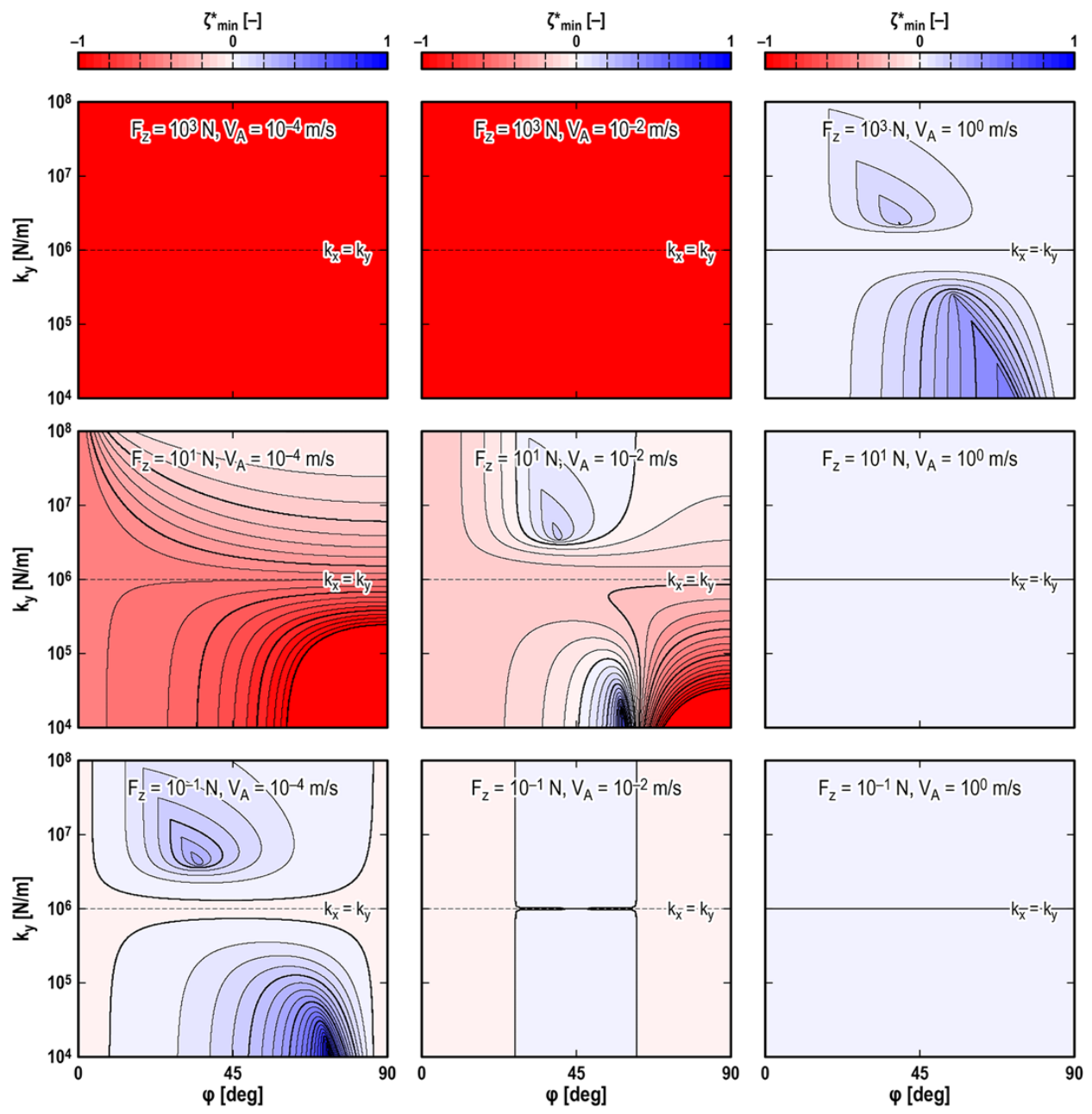

Fig. 6 Stability maps in $k_{y}-\varphi$ plane with minimum modal damping ratio $\zeta^{*}{ }_{\min } ; m=1 \mathrm{~kg}$, $k_{x}=10^{6} \mathrm{~N} / \mathrm{m}, \mu_{\mathrm{k} 0}=2, \mu_{\mathrm{k} \infty}=1$, and $V_{\mathrm{f}}=10^{-2} \mathrm{~m} / \mathrm{s}$; red: unstable $\left(\zeta^{*}{ }_{\min }<0\right)$ and blue: stable $\left(\zeta_{\min }^{*}>0\right)$

Only the red color $\left(\zeta^{*}\right.$ min $<0$ : unstable) can be seen in the top-left, top-middle, and middle-left graphs, which means that any combination of $k_{y}$ and $\varphi$ within the graph cannot stabilize the sliding system. However, in the rest six graphs, the blue color $\left(\zeta^{*}{ }_{\min }>0\right.$ : stable) can be seen. Especially, in every graph located on the diagonal from the bottom- 
left to the top-right, two deep blue portions surrounded by dense contour lines can be seen above and below the horizontal broken lines. By considering the positions of the deep blue portions, for example, a combination " $k_{y} \sim 10^{7} \mathrm{~N} / \mathrm{m}$ and $\varphi \sim 40^{\circ}$ " or another combination " $k_{y} \sim 10^{4} \mathrm{~N} / \mathrm{m}$ and $\varphi \sim 50^{\circ}$ " seems to be favorable for stabilizing the sliding system.

Note that the sliding system is unstable on the horizontal line $\left(k_{x}=k_{y}\right.$ : isotropic) and the two vertical lines $\left(\varphi=0^{\circ}\right.$ and $\varphi=90^{\circ}$ : symmetric), although the bottom-right graph seems to be filled with the blue color. For example, the "H-shaped" red area observed in the bottom-left graph is the area spread from the three unstable lines.

Friction-induced instabilities have been often discussed with the conventional 1DOF sliding model [10]. It is a picture when we see the $2 \mathrm{DOF}$ sliding system of $\varphi=0$ in the "front view" (i.e., in the $y$ direction). In other words, it is implicitly assumed that the sliding model is isotropic and symmetric. However, based on the stability maps proposed in this study, we realize that the conventional 1DOF sliding model just have provided the discussion on the horizontal broken line of the left graph in Fig. 5 or the discussion on the vertical line $\varphi=90^{\circ}$ of the graphs in Fig. 6. Now we can discuss the instabilities caused by the velocity-weakening friction based on the stability maps. If we accept the mechanical structure design of sliding systems with the in-plane anisotropy $\left(k_{x} \neq k_{y}\right)$ and the in-plane asymmetry $(\varphi \neq 0)$, we can find new stable conditions spread widely in the two-dimensional space, which are invisible from the conventional point of view.

\section{CONCLUSION}

The stability of the 2DOF sliding system (Fig. 1) was examined by the eigenvalue analysis, with considering the in-plane anisotropy $\left(k_{x} \neq k_{y}\right)$ and the in-plane asymmetry $(\varphi \neq$ 0 ). The results were organized by using the minimum modal damping ratio $\zeta^{*}{ }_{\min }$ (Eq. (32)) as the stability maps (Figs. 5 and 6). Especially for the stability maps in the $k_{y}-\varphi$ plane (Fig. 6 ), selecting a combination of the two quantities $\left(k_{y}\right.$ and $\varphi$ ) from the deeper blue portions corresponds automatically to embedding the YAM method in the mechanical structure design to avoid instabilities caused by the velocity-weakening friction.

Again, when engineers have to solve friction-induced instability problems, we must not forget that they always have two types of options. As a guideline for "improving mechanical structures", the authors believe that the stability maps considering the in-plane anisotropy and the in-plane asymmetry can be powerful tools, which would at least reduce the effort for "improving frictional properties" involving the trial-and-error processes. If we accept the mechanical structure design of sliding systems with the in-plane anisotropy and the in-plane asymmetry, we can find new stable conditions spread widely in the twodimensional space, which are invisible from the conventional point of view.

\section{REFERENCES}

1. Den Hartog, J.P., 1956, Mechanical Vibrations (Fourth Edition), McGraw-Hill, New York.

2. Kado, N., Tadokoro, C., Nakano, K., 2013, Measurement error of kinetic friction coefficient generated by frictional vibration, Transactions of the Japan Society of Mechanical Engineers, Series C, 79, pp. 2635-2643.

3. Kado, N., Tadokoro, C., Nakano, K., 2014, Kinetic friction coefficient measured in tribotesting: influence of frictional vibration, Tribology Online, 9(2), pp. 63-70. 
4. Nakano, K., Tadokoro, C., Kado, N., 2013, Yawing angular misalignment provides positive damping to suppress frictional vibration: basic applicability to disc brake systems, SAE International Journal of Passenger Cars: Mechanical Systems, 6, pp. 1493-1498.

5. Kado, N., Sato, N., Tadokoro, C., Skarolek, A., Nakano, K., 2014, Effect of yaw angle misalignment on brake noise and brake time in a pad-on-disc-type apparatus with unidirectional compliance for pad support, Tribology International, 78, pp. 41-46.

6. Tadokoro, C., Nagamine, T., Nakano, K., 2018, Stabilizing effect arising from parallel misalignment in circular sliding contact, Tribology International, 120, pp. 16-22.

7. Kado, N., Nakano, K., 2017, Stabilizing effect of in-plane angular misalignment in 2DOF sliding system with in-plane anisotropic stiffness, Mechanics Research Communications, 84, pp. 14-19.

8. Benad, J., Popov, M., Nakano, K., Popov, V.L., 2018, Stiff and soft active control of friction by vibrations and their energy efficiency, Forschung im Ingenieurwesen, 82(4), pp. 331-339.

9. Benad, J., Nakano, K., Popov, M., Popov, V.L., 2019, Active control of friction by transverse oscillations, Friction, 7(1), pp. 74-85.

10. Nakano, K., 2006, Two dimensionless parameters controlling the occurrence of stick-slip in a 1-DOF system with Coulomb friction, Tribology Letters, 24(2), pp. 91-98. 